\title{
Kangaroo Mother Care Practices: Sustainability Is Important
}

\author{
Anu Thukral ${ }^{1}$ \\ Received: 26 March 2021 / Accepted: 13 April 2021 / Published online: 20 April 2021 \\ (C) Dr. K C Chaudhuri Foundation 2021
}

Kangaroo mother care (KMC) is an evidence-based cost-effective approach which has benefits beyond immediate newborn survival including healthy growth and long-term development $[1,2]$. KMC with near-universal coverage can avert up to 450,000 preterm deaths every year [3]. Potential barriers to $\mathrm{KMC}$ implementation and coverage include issues with facility resources and environment, health care provider's attitude, and their lack of awareness about KMC benefits.

It is truly remarked that 'one size fits all' approach cannot guide $\mathrm{KMC}$ implementation. KMC is a complex intervention and each unit has its unique barriers and enablers which decide its uptake. Shifting healthcare provider beliefs, improving parental presence, identifying creative solutions to overcome space constraints, and development of clear guideline for KMC are some avenues which may provide impetus to change practices and overcome barriers [4].

Two recent studies suggested an improvement in $\mathrm{KMC}$ duration $[5,6]$ through a quality improvement (QI) approach in a tertiary-care neonatal unit. A QI team was formed, solutions were prioritised through focus group discussions and implemented through Plan-Do-Study-Act (PDSA) cycles. While the first study suggested that the duration of KMC improved [5], the second study also suggested an improvement in the timing of initiation of KMC. The average day of initiation of KMC improved from 8th to 3rd day of life [6].

In this issue of the Journal, the study by Kapoor et al. included one-to-one intensive counseling of mother and residential elderly woman (REW) on the benefits and procedure of KMC [7]. A brief $10 \mathrm{~min}$ video film, pictorial brochures, and focussed group discussion formed a part of this educational package. The intervention resulted in early initiation of $\mathrm{KMC}$ and increased duration of KMC. It increased proportion

Anu Thukral

dranuthukral@gmail.com

1 Department of Pediatrics, All India Institute of Medical Sciences, New Delhi 110029, India of neonates receiving $\mathrm{KMC}$ in the unit and at home after discharge and also improved exclusive breast-feeding rates.

While, intensive counseling and education, increasing support from staff is known to scale up KMC practices, the effect may be transient and fade away after withdrawal of support $[8,9]$. It is important to see the sustenance of the gains beyond the study period. Other outcomes like maternal satisfaction, sleep deprivation due to frequent milk expressions (median approximately 8 expressions per mother per day in intervention period in the index study) and staff feeling overworked due to intensive one-to-one counseling are other parameters which would be important to look at. This is important because utilization of existing resources, facility infrastructure and staff satisfaction and involvement without feeling overworked are important determinants for long-term sustenance of any quality improvement work. Audit-and-regular feedback mechanism is yet another step for changing health worker behavior in addition to an ongoing policy formation for sustenance of quality improvement work in the unit. Overall, the study did demonstrate that simple KMC education protocol is feasible and results in improved outcomes in preterm neonates.

\section{Declarations}

Conflict of Interest None.

\section{References}

1. Charpak N, Tessier R, Ruiz JG, et al. Twenty-year follow-up of kangaroo mother care versus traditional care. Pediatrics. 2017;139(1):e20162063.

2. Boundy EO, Dastjerdi R, Spiegelman D, et al. Kangaroo mother care and neonatal outcomes: a meta-analysis. Pediatrics. 2016;137:2015238.

3. Engmann C, Wall S, Darmstadt G, Valsangkar B, Claeson M. Participants of the Istanbul KMC Acceleration Meeting. Consensus on Kangaroo mother care acceleration. Lancet. 2013;382(9907): e26-7. 
4. Coutts S, Woldring A, Pederson A, De Salaberry J, Osiovich H, Brotto LA. What is stopping us? An implementation science study of kangaroo care in British Columbia's neonatal intensive care units. BMC Pregnancy Childbirth. 2021;21(1):52.

5. Joshi M, Sahoo T, Thukral A, Joshi P, Sethi A, Agarwal R. Improving duration of kangaroo mother care in a tertiary-care neonatal unit: a quality improvement initiative. Indian Pediatr. 2018;55(9):744-7.

6. Arora P, Kommalur A, Devadas S, Kariyappa M, Rao SPN. Quality improvement initiative to improve the duration of kangaroo mother care for twin preterm neonates born at a tertiary care hospital in resource-limited settings. J Paediatr Child Health. 2021 Feb 26. https://doi.org/10.1111/jpc.15406.

7. Kapoor R, Verma A, Dalal P, Gathwala G, Dalal J. Enhancing kangaroo mother care uptake through implementation of an education protocol. Indian J Pediatr. 2020 Oct 20. Doi: https://doi.org/10.1007/ s12098-020-03537-z. Online ahead of print

8. Bergh A-M, Kerber K, Abwao S, et al. Implementing facility-based kangaroo mother care services: lessons from a multicountry study in Africa. BMC Health Serv Res. 2014;14:293.

9. Kirigia JM, Nganda BM, Mwikisa CN, Cardoso B. Effects of global financial crisis on funding for health development in nineteen countries of the WHO African region. BMC Int Health Hum Rights. 2011;11:1.

Publisher's Note Springer Nature remains neutral with regard to jurisdictional claims in published maps and institutional affiliations. 